\title{
THE MACROMOLECULAR ORGANIZATION OF MEMBRANES AND ITS BEARING ON EVENTS LEADING UP TO FERTILIZATION
}

\author{
M. H. JOHNSON \\ Department of Anatomy, University of Cambridge, Downing Street, Cambridge
}

\begin{abstract}
Summary. Current concepts of the macromolecular organization of membranes and of ways in which membrane organization may change during membrane fusion are considered briefly. The properties of the sperm membrane at different phases of its development up to fertilization are then examined in the light of this more general model.
\end{abstract}

Membrane fusion and changes in membrane properties evidently occupy a fundamental rôle in the process of fertilization, yet our knowledge of the membrane properties of the egg and spermatozoon are still rudimentary. This review will outline the concepts currently held about general membrane properties and will attempt to relate observations on gamete membranes to a more general framework.

\section{THE STRUCTURE OF MEMBRANES}

In considering the events involving fusion of intracellular organelles such as the acrosome or cortical granules with the plasma membrane, the basic supposition will be made that some perturbation of the previously existing organization of macromolecules in the membranes concerned and some fluidity in the membrane are essential prerequisites. Such changes may possibly occur in response to changes in the internal milieu, as indicated in the previous paper. Alternatively, stimuli for fusion in the acrosome reaction and the release of cortical granules may come from events external to the cell and so would need to be transmitted internally in some way. There are a number of ways in which the macromolecular reorganization might be envisaged. For example, a local transition of membrane lipids from linear arrays of bimolecular leaflets into micellar organization might be important (see Lucy, 1975). Another possibility is the reorganization of intramembranous macromolecules and lipids into local aggregations or domains. These two possibilities are not, of course, mutually exclusive or necessarily comprehensive.

A visually beautiful example of the way in which membrane macromolecules may reorganize at fusion has been described by Satir and her colleagues (Satir, Schooley \& Satir, 1972, 1973) during the discharge of mucocysts in the ciliated protozoan Tetrahymena pyriformis. In this process, clusters of particles within the cytoplasmic membrane of freeze-fractured cells form in apposition to similar 
clusters in the cytoplasmic face of the mucocyst membrane. The organelle then fuses with the surface at this point. Similarly, formation of the pinocytotic vacuole in rat lung epithelial cells involves redistribution and clustering of intramembranous particles at the site of fusion (Smith, Ryan \& Smith, 1973). Conditions under which Sendai virus and dimethylsulphoxide induce fusion of erythrocytes also stimulate clustering of intramembranous particles (Bächi \& Howe, 1973). Whilst the more obvious common feature of these observations is the clustering of intramembranous particles which are probably of glycoprotein nature, it is entirely possible that this process is associated with changes in lipid microenvironments which may be of equal or greater significance for the fusion events. It is pertinent then to consider the organization of macromolecules and lipids in non-fusing membranes and how the organization of these molecules might be modified in response to internal change or external stimuli.

The model of the fluid mosaic membrane proposed and developed over the last few years by various workers (see Singer, 1971; Singer \& Nicolson, 1972) has found broad support on a number of lines of evidence. The bulk of the membrane is held to be a matrix of phospholipids organized in the traditional bimolecular leaflet fashion of the Davson-Danielli-Robertson model (see Singer, 1971), but with a substantial part of the structural and functional proteins integral to the membrane intercalated to greater or lesser degrees into the lipid bilayer itself. These so-called 'intrinsic' proteins have polar residues protruding into the aqueous hydrophilic regions on one or both sides and non-polar hydrophobic residues within the membrane adjacent to the fatty acid chains. Integral to the normally functional membrane of the cell, although not essential to its structure, are the so-called 'extrinsic' macromolecules that are applied relatively loosely by polar bonds to the external surface of the membrane, and other components, notably microfilaments and microtubules, attached to the internal surface.

It has been demonstrated in a variety of ways that some macromolecules are free to move within the plane of the membrane, as if floating in a sea of lipid. The lipids themselves can also move in relation to each other and to the protein. One of the first and most elegant demonstrations of the ability of some macromolecules to show relatively free lateral diffusion under some circumstances was by Frye \& Edidin (1970), who measured the intermixing of surface antigens in heterokaryons of mouse and human cells induced by inactivated Sendai virus. At intervals, portions of the cell suspension were reacted with fluoresceinlabelled antibodies to mouse $\mathrm{H}-2$ antigens and with rhodamine-labelled antibodies to human antigens. The shift from $100 \%$ of heterokaryons with separate red and green fluorescent hemispheres to $100 \%$ with intermingled red and green membranes took about $40 \mathrm{~min}$, was not sensitive to inhibitors of protein synthesis and oxidative phosphorylation, but was slowed by cooling. A variety of other studies have used electron spin resonance (ESR) to estimate the diffusional constants of spin-labelled lecithin or steroid molecules introduced into artificial or extracted natural membranes, either by analysing the ESR line shape of the label under different conditions of temperature or membrane composition, and thereby inferring ease of interaction of spin-labelled molecules (Träuble \& Sackmann, 1972; Scandell, Devaux \& McConnell, 1972), or by 
recording the broadening with time of ESR signals from the concentration of label in a spot of pure probe (Devaux \& McConnell, 1972). This latter measure gives an indication of how readily the probe molecules can diffuse laterally, thus diluting the concentration of the probe. Several other studies using tagged proteins, ESR and nuclear magnetic resonance have been useful in obtaining estimates for the freedom with which molecules move within artificial and natural membranes under different conditions. Reported estimates of diffusional constants for different systems have recently been collated by Edidin (1974) and shown to lie within approximately a thirty-fold range of one another. Whilst details of those measurements need not concern us here, the important conclusion is that under appropriate conditions lateral diffusion of membrane components can and does occur.

Factors influencing the facility with which macromolecules may move within the membrane, and more specifically tending to hold membrane organization in a less mobile state, next require attention. These considerations come under two broad headings; factors influencing the solvent or lipid phase, and those affecting the intramembranous macromolecules themselves.

\section{The lipid phase}

Temperature has effects on lateral diffusion of molecules in membranes, but the relationship between temperature and diffusion rates need not be simple. As membranes of pure lipid are cooled or warmed they go through a phase transition, in which the lipids essentially 'freeze' or 'thaw'. Such changes can be measured directly by the heat transfers involved, or indirectly by the changes in mobility of lipids or proteins recorded. In more complex membranes composed of several lipids, with different gelling points, phase separation of lipids may occur, segregating the membrane into regions of relatively high ('gel') and low ('sol') viscosity areas. Recently, Petit \& Edidin (1974) have shown that the rate of mixing of surface antigens on newly formed mouse-human heterokaryons is retarded by cooling cells from $37^{\circ} \mathrm{C}$ to $21^{\circ} \mathrm{C}$, but then speeded up by further cooling to $15^{\circ} \mathrm{C}$. They offer an explanation for this secondary effect as being due to segregation of the lipid phase of the membrane into islands of solid lipid from which antigens are excluded, and areas of liquid phase now at a lower viscosity. The possibility that membranes of mixed lipid phases could occur at physiological temperatures is real, and several factors would influence the state of such membranes. For example, membranes containing short-chain unsaturated fatty acids, show a lower phase-transition temperature. Selective incorporation of the desired fatty acids into membranes has been achieved by regulation of the fatty acid supply in cultures in which Escherichia coli or mouse cells are grown and the membrane properties of the cells were thereby affected (Machtiger \& Fox, 1973; Wisnieski, Williams \& Fox, 1973). Introduction of cholesterol into the membrane smooths out the sharp phase-transition effect. The cholesterol seems to render a gel phase more sol, and a sol phase more gel (Chapman, 1972; Shimshick \& McConnell, 1973). These effects of lipids on membrane stability, like those of lysolecithin and hyamine (see Lucy, 1975; Wooding, 1975) can probably best be thought of as working by introducing an irregularly shaped molecule into the lipid phase of membrane, thus affecting 
the closeness with which molecules may pack. These effects of fatty acid composition and cholesterol on the physical properties of membranes have important influences on the metabolic and physiological function of cells and organelles in vivo and in vitro (Overath, Hill \& Lamneh-Hirsch, 1971 ; Linden, Wright, McConnell \& Fox, 1973). Thus, the transfer of $\beta$-galactoside into $E$. coli has been shown to be critically dependent upon the membrane lipid being in the sol rather than the gel phase (Overath et al., 1971). A direct visualization of the effects of temperature has come from freeze-fracturing studies on particles in Mycoplasma membranes (Tourtellotte, Branton \& Keith, 1970). Gells grown in medium containing non-saturated oleic acid showed a scattered particle array after snap-freezing at room temperature, whereas those grown in the saturated stearic acid showed clustered particles with smooth membrane between, suggesting there might be areas from which particles were excluded. The random array seen in the membranes of Mycoplasma grown in oleic acid could be converted to a clustered distribution by cooling prior to freeze-cleaving.

The phase transition of lipids in membranes is accompanied by changes in the distribution of charge at the surface of the phospholipid. This results from the increased packing of lipids in the gel phase, in which state is observed an increased ionization and hydration of polar groups. Conversely, changes in the ionic environment of the polar groups of lipids affect the phase-transition temperature. Thus, Träuble \& Eibl (1974) have shown that the phasetransition temperatures of various lipid bilayers can be greatly influenced by rather small changes in $\mathrm{pH}$ or ionic composition. An increase in the concentration of divalent cations such as $\mathrm{Ca}^{++}$, for example, raises the melting point by neutralizing surface charges and facilitating closer packing of phospholipids at any given temperature.

To summarize, then, the mobility of macromolecules within the membrane is affected, and could possibly be directed, by the lipid phase of the membrane, either over the cell membrane as a whole, or, where surface mosaicism is evident, in defined microenvironments or limited areas of cell membrane. The sorts of factors known to influence the lipid phase are temperature, fatty acid and cholesterol composition, $\mathrm{pH}$ and ionic environment. There is considerable evidence that a fluid array of lipids will be more conducive to fusion, or even essential for it.

\section{Intrinsic proteins}

The movement of macromolecules will be affected not only by the solvent medium in which they can move, but by their own molecular bulk, the degree to which they are embedded in the lipid bilayer and by their interactions with each other and with anchoring or restraining mechanisms applied from inside or outside the membrane. Such mechanisms could constitute one explanation for the molecular organization underlying the observed mosaicism or polarity of function in many cells. That organized clustering occurs can be seen in many instances, e.g. the mucocyst and surface intramembranous particles in Tetrahymena discussed above. Another example is the septate junction or desmosome between electrically-coupled cells of the mussel gill epithelia, in which arrays of $85 \AA$ particles in each cell align on either side of the material in the junctional 
septa (Gilula, Branton \& Satir, 1970), perhaps held there by the material of the junctional septa. Similar clusterings presumably occur at most organized cellcell junctions, but when cells are disaggregated into single cell suspensions, the intramembranous particles disperse in the membrane.

In all of these studies just mentioned, it seems clear that the clustering of particles is a genuine reflection of the state of organization of the membrane. There is, however, an onus on investigators to prove this point, since studies on several cell types have shown that clustering can be induced by various procedures (see Munro, 1975). For example, erythrocyte ghosts possess randomly distributed $85 \AA$ particles bearing blood-group antigens and influenza virus receptors. On treatment of the membranes with trypsin, the particles are found in clusters. Trypsin exerts a similar effect on the Concanavalin A (Con A) binding sites of murine 3T3 fibroblasts (Nicolson, 1972). Clustering on erythrocyte ghosts also occurs with low $\mathrm{pH}$, but is prevented by high ionic strength. Sendai virus induces particle clustering in red cells before their fusion, possibly by the action of neuraminidase (Bächi \& Howe, 1973). It has been suggested that proteolytic enzymes may act by reducing the surface charge or hydrophilicity of the exposed surface of the particles, either by removal of parts of the molecule itself or possibly by removal of an overlying surface protein. Desialation by neuraminidase might act similarly. With the reduced surface charge, particles would be less mutually repellent, and less repellent to adjacent apposed membranes (Poste \& Allison, 1973).

Aggregation of intramembranous macromolecules or particles can also be induced by application of external cross-linking agents, such as antibodies or plant lectins. The requirements are that the cross-linking agent and the intramembranous macromolecule both be at least divalent, and that there be molecular mobility in the membrane. An energy-independent, but temperaturedependent aggregation of macromolecules into patches occurs, much like a two-dimensional precipitation in the membrane. If an energy source is available to the cell, then the patches may be gathered up into a cap at one end of the cell (see Munro, 1975). Both patching and capping by antibodies may evidently induce phagocytosis, again involving membrane fusion. Cross-linking of surface macromolecules, for example, has been shown to influence other organelles within the cell and, of significance in connection with the acrosome and cortical granule, the stability of lysosomal membranes (Allison \& Malluci, 1964; Hirschhorn, Brittinger, Hirschhorn \& Weissmann, 1968; König, Brittinger \& Cohnen, 1973). A direct effect of the cross-linking on isolated lysosomes could not be detected; somehow the surface perturbation was recorded internally, and had an effect. The success of cross-linking agents in modifying macromolecular organization will depend on the bonds linking them to intrinsic molecules being strong enough to overcome the resistance to movement of the molecule in the membrane. Thus, the coating of diverse classes of extrinsic macromolecules overlying the basic membrane might theoretically be in a position to stabilize membrane organization depending on their affinity of binding for intrinsic proteins, and changes of these coating layers could well result in changes of membrane organization or stability.

The changes effected in the properties of internal organelles, such as lyso- 
somes, or in formation of phagocytic vesicles, seem to involve intracellular proteins which may also show attachment to, and cross-linking of, the internal aspects of intramembranous macromolecules. Pinocytosis, capping and some types of secretion are sensitive to cytochalasin B, which, among other properties, probably inhibits the globular to fibrillar conversion of the actin-like microfilaments lying just beneath, and attached to, the surface membrane. For example, the uptake and release of a number of CNS transmitter substances has been found to be greatly reduced in the presence of cytochalasin B (Nicklas \& Berl, 1974). The contraction of microfilaments seems to require $\mathrm{Ca}^{++}$, for which $\mathrm{Sr}^{++}$or $\mathrm{Ba}^{++}$, but not $\mathrm{Mg}^{++}$, will substitute (Allison, 1973).

A more extensive internal cross-linking system associated with the microtubule system of the cell has been postulated by Edelman, Yahara \& Wang (1973) on the basis of studies on the effect on the plant lectin Con A on lymphocytes. Con A at large enough doses causes such extensive cross-linking at the cell surface that the whole mobility of all intramembranous macromolecules is frozen. The effect can be reversed by performing the cross-linking at low temperatures with subsequent warming of the cell, or by treating the cell with colchicine, colcemid or vinblastine. Edelman and his colleagues postulate the existence of an intracellular colchicine-binding, temperature-sensitive protein assembly that is capable of immobilizing the macromolecules in the intact membrane in response to excessive cross-linking. In normal circumstances it is possibly this system which brings about selective stability or organization of macromolecules within the membrane and which offers at least one route by which surface active or surface cross-linking agents might influence intracellular events. As an example of the possible role of this system, Ukena \& Berlin (1972) have found that during phagocytosis by leucocytes involving up to $50 \%$ of the surface membranes, receptors mediating transmembrane transport were not ingested unless colchicine was added. Possibly the internal colchicine-sensitive protein normally held the receptors in the membrane as it flowed round them into phagosomes. Redistribution of surface groups in transformed cells under different conditions may also involve such a system (Inbar, Ben-Bassat \& Sachs, 1972).

Despite the brevity of this outline, it is clear that the results of considerable numbers of experiments demonstrate that external and internal cross-linking agents can play an important role in moving and stabilizing intramembranous macromolecules. This conclusion has obvious relevance to mechanisms of membrane fusion in general and to gametes at fertilization in particular. The critical questions we therefore need to answer about gamete physiology concern the degree of mobility or fluidity of the lipid and macromolecular components under different conditions. Such fluidity seems to be a prerequisite for fusion (Howell, Ahkong, Cramp, Fisher, Tampion \& Lucy, 1972; Papahedjopoulos, Poste \& Shaeffer, 1973).

\section{SPERM MATURATION}

Despite considerable literature on factors controlling or influencing fertilization, there is remarkably little information about the macromolecular structure and 
organization of gamete membranes, the changes in this organization in different maturational states, or the factors that influence organization critically in any given state. This situation arises largely because most investigators, often through experimental necessity, have tried to 'optimize' conditions for several sequential steps of sperm development rather than analysing the optimal conditions for each serial step. For spermatozoa, analysis of at least six membrane phases is necessary; namely, the testicular, epididymal, capacitated, activated (which I shall take to include acrosome-reacted), attached and fused phases. It is important to emphasize the distinction between capacitation, which is the process of nonvisible change normally undergone by spermatozoa in the female tract, from the subsequent activation, comprising the acrosome reaction and whiplash tail movement. These processes are functionally distinct although the one in physiological terms is an essential precondition of the other. In the following account, the spermatozoon will be followed in its passage through the genital tracts, and the limited data available on the changes in membrane properties will be discussed.

The fact that testicular spermatozoa will not manifest any of the postcapacitation properties testifies to the importance of epididymal maturation for membrane changes. Differences in the lipid composition of testicular and ejaculated ram spermatozoa have been studied in some detail by Scott, Voglmayr \& Setchell (1967), and from these results three features emerge which may be of some significance for membrane maturation.

First, testicular spermatozoa have higher lecithin levels than ejaculated spermatozoa, and the phase-transition temperature of lecithin is known to be less affected by ionic change than other lipids. Second, the cholesterol content of ejaculated spermatozoa is half that of testicular spermatozoa, and as was discussed earlier, cholesterol tends to smooth out phase-separation or transition effects and stabilize the membrane. Third, the ratio of saturated to unsaturated fatty acids in testicular spermatozoa is higher than that for ejaculated spermatozoa, and unsaturated fatty acids tend to lead to lower phase-transition temperatures and greater membrane fluidity. All three features suggest that the ejaculated spermatozoa might possess less stable or more 'fluid' membranes. The location of the changes in lipid composition are not known. An organized mosaicism of lipid distribution has been described for the inner and outer layers of the bimolecular leaflets of the erythrocyte membrane, so it is entirely possible that the maturational lipid changes of the spermatozoon could be concentrated in one limited area of the highly heterogeneous sperm membrane (see below), with profound effects on local membrane stability. Use of fluorescent probes such as 1-anilino-naph thalene-8-sulphonate (ANS) or ESR to investigate the constraints applied to hydrophobic regions of whole or fractionated spermatozoa taken from different levels of the male genital tract might provide useful data on this point. Introduction of fatty acids labelled with paramagnetic reporter groups into ejaculated rabbit spermatozoa has been described (Hsia, Piette \& Noyes, 1969), with obvious immobilization of the spin-labelled molecules, but comparison of testicular, epididymal and capacitated spermatozoa was not undertaken.

The plasmalemma of the spermatozoon shows ample evidence of mosaicism 
of macromolecular structure. Some of the intrinsic macromolecules of sperm membranes possess terminal saccharide surface groups reactive with a variety of plant lectins. Fluorescent Con A applied to guinea-pig spermatocytes and spermatids gives bright surface staining. On testicular spermatozoa the staining is weaker, predominantly overlying the acrosome but with some weak fluorescence on the rest of the sperm membrane. This staining is blocked or reduced by $\alpha$-methyl-D-mannoside and is smooth and continuous, not patchy in distribution (M. H. Johnson, unpublished data). A similar result for epididymal mouse spermatozoa was reported by Edelman \& Millette (1971). Agglutination studies on rabbit and hamster epididymal spermatozoa further suggest a primarily acrosomal plasma membrane location for the Con A receptors, and characteristic but different agglutination patterns occur for other plant lectins and for influenza virus which binds to sialic acid residues on the acrosomal membrane (Nicolson \& Yanagimachi, 1972). A mosaicism in the surface properties of the membrane of epididymal spermatozoa is further indicated by the selective binding of colloidal iron hydroxide to negative sites on the sperm tail and neck but not to the post-acrosomal region and only weakly to the acrosomal region in several but not all species (Cooper \& Bedford, 1971; Yanagimachi, Noda, Fujimoto \& Nicolson, 1972; Yanagimachi, Nicolson, Noda \& Fujimoto, 1973). The negative charge on the tails was not neuraminidase-sensitive, which correlates with the failure of influenza virus to bind to sperm tails. Lindahl (1973) has described a $\mathrm{Ca}^{+++}-\mathrm{ATP}$ dependent agglutination of washed bull spermatozoa which occurs solely between the tips of the acrosomal plasmalemma. The integral sperm antigen, designated $T$ by Voisin and his colleagues, has been detected and quantified on spermatids, testicular and epididymal spermatozoa (Johnson, 1970; Toullet \& Voisin, 1974), and is localized on the head and midpiece but not on the tail mainpiece (Johnson \& Edidin, 1972). In contrast, the $\mathrm{Y}$ antigen appears to be limited to the region of the acrosomal plasma membrane (Koo, Stackpole, Boyse, Hammerling \& Lardis, 1973).

By far the most important study on membrane mosaicism in spermatozoa however is that of Nicolson \& Yanagimachi (1974) using the ferritin-conjugated agglutinins from Ricinus communis to probe not only the distribution but also the relative mobility of $D$-galactose residues in different regions of the membranes of rabbit epididymal spermatozoa. After incubation at $0^{\circ} \mathrm{C}$, continuous labelling over the whole spermatozoal membrane was found. But if incubation was performed at $37^{\circ} \mathrm{C}$, clustering of marker molecules was observed in the postacrosomal region but not over the bulk of the tail membrane or over the acrosomal plasma membrane. Using fluorescent Con $\mathrm{A}$ and fluorescent antibodies at $\times 100$ magnification, we have also failed to detect patching on the acrosomal surface membranes, despite being able to clearly identify this phenomenon on other cells such as cleaving blastomeres, trophoblast or lymphocytes under these conditions (Johnson, 1975, and unpublished data). At the electron microscopic level, Koehler \& Perkins (1974) were also unable to detect mobility of heteroantigens present on the acrosomal cap. The constraint on the movement of receptors in the surface membranes overlying the acrosome could be an apparent effect arising from trivial causes but the more interesting possibility is that the constraint is imposed perhaps by internal or by external cross-linking 
or by a lack of fluidity of the membrane lipid. We do not yet know the cause or whether the constraint occurs at all levels of the male tract.

Several studies thus combine to suggest a rather complex surface mosaicism of both the structure and fluidity of the sperm membrane. Comparison of testicular and epididymal spermatozoa has shown that there is little qualitative change in the mosaic pattern of the surface membrane; any changes that do occur appear to affect either the whole surface membrane or to exaggerate the existing mosaicism. For example, Con A binding seems to rise marginally (unpublished data), the total surface negative charge seems to increase (Bedford, 1963; Yanagimachi et al., 1972) and the ability of the acrosomal membrane to bind Sendai virus decreases (Ericsson, Buthala \& Norland, 1971) during passage of spermatozoa through the epididymis. Similar changes affecting the quantitative rather than qualitative features of macromolecular distribution are seen on comparing epididymal and ejaculated spermatozoa. Thus, the binding of wheat-germ agglutin to $\mathrm{N}$-acetyl-D-glucosamine residues on both head and tail is evidently increased after ejaculation (Nicolson \& Yanagimachi, 1972). A number of studies have reported the presence of glycoprotein macromolecules, the so-called coating antigens, in the seminal plasma and epididymal secretions that seem to bind with considerable affinity over the whole sperm surface (Chang, 1957; Bedford \& Chang, 1962; Weil \& Rodenburg, 1962; Weinman \& Williams, 1964; Dukelow, Chernoff, Pinsker \& Williams, 1966; Dukelow, Chernoff \& Williams, 1966, 1967a, b; Pinsker \& Williams, 1967; Hunter \& Nornes, 1969; Johnson \& Hunter, 1972a; Killian \& Amann, 1973). As the whole sperm membrane is involved in the post-capacitation changes, it is not surprising that comparative studies have stressed overall effects over the sperm membrane regardless of its underlying macromolecular mosaicism. A major omission has been the lack of study of the functional state of membrane mobility in the different maturational stages.

\section{GAPAGITATION}

This omission is also seen in studies on capacitation. It is established that many of the coating antigens are removed again in the female tract. For example, the Con A binding to the surface of the rabbit spermatozoon becomes weaker after incubation in the uterus (Gordon, Dandekar \& Bartoszewicz, 1974), and the increase in negative charge seen during epididymal maturation is reversed, possibly due to loss of sialic acid residues (Vaidya, Glass, Dandekar \& Johnson, 1971; Rosado, Velazquez \& Lara-Ricalde, 1973). No morphological changes have been detected in the sperm membrane at the ultrastructural level, although Yanagimachi \& Usui (1974) reported slight fuzziness on the surface. Considerable literature exists on the release of the extrinsic macromolecules in incubation conditions in vivo or in vitro (Kirton \& Hafs, 1965; Ericsson, 1967; Hunter \& Nornes, 1969; Vaidya, Bedford, Glass \& Morris, 1969; Johnson \& Hunter, 1972b; Aonuma, Mayumi, Suzuki, Noguchi, Iwai \& Okabe, 1973; Oliphant \& Brackett, 1973a). The elution of these extrinsic proteins in simple defined media over a period of hours might be expected, due to equilibrium adjustment. This process would be hastened by the higher ionic strength of tissue culture 
media compared with that of male genital tract fluids, in which organic molecules constitute striking and important components of the osmolality as shown in Table 1 (Mahi \& Yanagimachi, 1973; Miyamoto \& Chang, 1973;

Table 1. Relative contributions to osmolality of fluids taken from the male genital tract of the rat

\begin{tabular}{lccc}
\hline Source of fluid & $\begin{array}{c}\text { Total } \\
\text { osmolality } \\
\left.\text { (mosmol/kg } \mathrm{H}_{2} \mathrm{O}\right)\end{array}$ & $\begin{array}{c}\text { Osmolality } \\
\text { contributed by } \\
\mathrm{Na}^{+} / \mathrm{K}^{+} / \mathrm{Cl}^{\prime} / \mathrm{HCO}_{\mathbf{3}}\end{array}$ & $\begin{array}{c}\text { Osmolality } \\
\text { contributed by } \\
\text { organic molecules }\end{array}$ \\
\hline Seminiferous tubules & 338 & 296 & 42 \\
Caput epididymidis & 315 & 147 & 168 \\
Corpus epididymidis & 340 & 109 & 231 \\
Cauda epididymidis & 329 & 91 & 238 \\
Ductus deferens & 339 & 73 & 256 \\
\hline
\end{tabular}

Adapted from Levine \& Marsh (1971).

Oliphant \& Brackett, 1973b). Glycerol, which has membrane fusion properties of its own, did not accelerate capacitation when tested for its osmotic effects (Oliphant \& Brackett, 1973b). Enzymatic activity evidently hastens the process of macromolecular elution as has been shown in vitro by the use of various enzymes such as pronase, trypsin, $\beta$-glucuronidase, $\beta$-amylase and neuraminidase (Kirton \& Hafs, 1965; Gwatkin \& Hutchison, 1971; Johnson \& Hunter, 1972b; R. Yanagimachi, personal communication) and is suggested in vivo by the advantageous capacitating environment provided by the oestrous uterus with its proteolytic activity (Joshi, Yaron \& Lindner, 1970; Joshi \& Murray, 1974) compared to the progestational uterus with protease inhibitors (Chang, 1951; Noyes, Walton \& Adams, 1958; Soupart, 1967; Hamner, Jones \& Sojka, 1968; Bedford, 1970; M. A. H. Surani, personal communication). The action of viral neuraminidase may be the basis for the efficacy of Sendai virus in causing entry into rabbit eggs by non-capacitated spermatozoa (Ericsson et al., 1971). In the testis, epididymis and semen the spermatozoa bear trypsin inhibitors which might assist resistance to removal of extrinsic proteins until the female genital tract is reached (Suominen \& Setchell, 1972). The one clue that suggests that these enzymatic influences on the spermatozoon affect the functional organization of its membranes comes from the observation that the quantum fluorescence yield of ANS increases after treatment of spermatozoa with neuraminidase, which would indicate that intramembranous proteins might have moved into a more hydrophobic microenvironment, perhaps by clustering (Mercado \& Rosado, 1973).

The relative contribution of enzymes and simple eluting conditions to the capacitation process seems to vary with different species. Thus, for rabbit spermatozoa, enzymatic treatment seems an essential prerequisite except under extreme conditions of ionic strength, although simple elution will assist normally (Johnson \& Hunter, 1972b), whilst for rodents, enzymatic treatment hastens 
but is not essential for capacitation. All of these observations testify to a considerable affinity of coating molecules for the sperm membrane.

These experimental observations are few, and it seems important to develop hypotheses to provide a framework for more rigorous investigations. A simple but attractive notion, for example, is that as the spermatozoa mature in the epididymis and are transported into the female tract their membranes become less stable, perhaps because of changes in lipid organization and the rise in ambient temperature, and therefore show an increasing need for stabilization during sperm storage and transport so as to avoid premature activation. The epididymal and seminal extrinsic factors might be seen as providing such stabilization either by providing an external excessive cross-linking system (Johnson \& Howe, 1975) or by maintaining an exaggerated surface repellent charge on intrinsic macromolecules. Capacitation could then be regarded as a progressive reduction in cross-linking or charge to a point where, given appropriate conditions, movement of macromolecules or lipids within the matured membrane becomes possible, leading perhaps to permeability changes and to membrane fusions. It is striking that elevated ionic strength, trypsin, Sendai virus and neuraminidase, all of which facilitate capacitation, are factors associated with clustering of intramembranous particles in other cells and such clustering is at least associated with membrane fusion if not directly implicated (see above). Such an hypothesis is open to test by various routes. The distribution of intramembranous particles in the acrosomal plasmalemma might differ in the capacitated, non-capacitated and decapacitated states, depending on whether spermatozoa are precooled before freeze-cleaving. A similar differential dependence on temperature of the labelling patterns with plant agglutinins or antibodies might be expected. In experiments using fluorescent Con $\mathrm{A}$ on capacitated non-activated guinea-pig spermatozoa, acrosomal patching could not be detected, but electron microscopic observations would provide a more sensitive and essential assay. Readdition of seminal or epididymal extrinsic coating factors to capacitated non-activated spermatozoa might be expected to restabilize or neutralize the membrane.

There is an extensive literature on decapacitation factors (DF) of disputed composition which are assayed by their ability to block the fertilization potential of capacitated spermatozoa and which are presumed to represent at least one of the extrinsic factors applied during sperm maturation (Dukelow et al., 1966; Pinsker \& Williams, 1967; Hunter \& Nornes, 1969; Robertson, Bhalla \& Williams, 1971). However, fertilization comprises several steps, any of which could be blocked irreversibly by DF without them truly being decapacitating. Surprisingly, there appear to have been no reported attempts either to visualize DF or to measure surface charge on recoated capacitated non-activated spermatozoa. The most decisive observations to suggest that DF do actually decapacitate are (a) that decapacitated spermatozoa require a full recapacitation period and are then fertile again (Bedford, 1970); (b) that capacitated spermatozoa which are treated with $\mathrm{DF}$ all have ultrastructurally normal and intact plasmalemmas in the presence of follicular fluid that was rapidly and effectively activating non-decapacitated spermatozoa (Bedford, 1970); (c) that DF are taken up from the medium during decapacitation; and (d) that radio- 
labelled antibodies to DF react with decapacitated spermatozoa (Oliphant \& Brackett, 1973a; Austin, 1975). These experiments suggest, but do not prove, that seminal and epididymal factors are actually restabilizing the plasmalemma, rather than killing the capacitated spermatozoa and thus necessitating the capacitation of other spermatozoa. A control in-vitro system which permits full capacitation but not activation of the vast majority of spermatozoa, such that addition of an activation factor will cause most to activate rapidly, would provide a means for decisively distinguishing these possibilities. Such a system has now been described and is discussed below.

The concept of capacitation as constituting a reduction in cross-linking or charge is not exclusive to other hypotheses. For example, the coating proteins might function by chelating divalent cations such as $\mathrm{Ca}^{++}$that are essential for activation (see below). The capacity of eluted coating proteins to be inhibitory in a $\mathrm{Ca}^{++}$titration assay might provide a useful experimental approach to test this hypothesis. Specific neutralization of enzymes might occur. In this context the anti-corona dispersing activity attributed to seminal components (Zaneveld \& Williams, 1970) would be more likely to affect activated spermatozoa. The suggestion by Gordon (1973) that coating substances might contain inhibitors of a surface ATPase involved in maintaining sperm function seems unlikely, since epididymal secretions do decapacitate but do not contain the inhibitor, and spermatozoa taken from the epididymis still require capacitation. Changes in membrane properties seem likely to occur during capacitation, but the inadequate data available permit only the most tentative proposals for an hypothesis regarding mechanisms.

\section{ACROSOME REACTION}

A recent advance which should prove of major significance in the study of conditions required for the capacitation changes in spermatozoa and their distinction from the post-capacitatory activation changes has been the complete separation of the two phases in vitro under controlled and therefore systematically variable conditions. Yanagimachi \& Usui (1974) have found that incubation of guinea-pig spermatozoa in simple modified Krebs-Ringer or Tyrode solutions containing $0.1 \%$ BSA results in approximately $60 \%$ of spermatozoa showing the acrosome reaction and the characteristic whiplash tail movement within 10 to $12 \mathrm{hr}$. If $\mathrm{Ca}^{++}$is omitted from the media, the post-capacitatory activation does not occur. On addition of $\mathrm{Ca}^{++}$to preincubated spermatozoa, however, the acrosome reaction and whiplash movement develop in large numbers of spermatozoa within minutes. Thus, the spermatozoa have been capacitated, presumably involving loss of coating antigens, but require $\mathrm{Ca}^{++}$to reveal the fact by activation. Strontium will replace $\mathrm{Ca}^{++}$, but $\mathrm{Mg}^{++}$will not. Levels of $\mathrm{Ca}^{++}$in excess of $10 \mathrm{~mm}$ inhibit the activation. These observations have been confirmed, and it has been demonstrated that the $\mathrm{Mg}^{++}$appears to counter the action of $\mathrm{Ca}^{++}$. Yanagimachi's group have shown that trypsin hastens capacitation but will not of itself cause the acrosome reaction. This elegant and extremely useful system provides a beautiful method of obtaining large numbers of capacitated but non-activated spermatozoa in vitro for analysis 
of the fluid membrane properties and the effect thereon of factors such as cross-linking agents, metabolic inhibitors, local anaesthetics, ions, and decapacitation factors.

The results obtained so far raise the question of the nature of the stimulus for the activation changes. $\mathrm{Ca}^{++}$is clearly a prerequisite, but it would be rash to assume that $\mathrm{Ca}^{++}$alone is sufficient. An essential role for albumin can probably be excluded, since Barros, Berrios \& Herrera (1973) have achieved capacitation plus activation in a saline-based medium free of macromolecules. However, a transient head-to-head agglutination of hamster spermatozoa by albumin might suggest some role for this macromolecule in the acrosome reaction phase (Yanagimachi, 1969). The capacity of albumin to sequester fatty acids and thus to affect the stability of membranes, particularly under conditions of increased fluidity suggests that a systematic analysis of the role of this molecule might be worthwhile. Such a consideration may be particularly important when making comparative investigations in a species such as the rabbit, in which it seems a factor in addition to divalent cations may be essential for activation. That $\mathrm{Sr}^{++}$ but not $\mathrm{Mg}^{++}$will substitute for $\mathrm{Ca}^{++}$suggests that the important property of $\mathrm{Ca}^{++}$is not simply divalency acting on the surface charge of the membrane to influence packing of phospholipids or aggregation of proteins. Again, however, a non-specific action of cations cannot necessarily be excluded as, for example, a precondition for a subsequent specific activity of $\mathrm{Ca}^{++}$. It might prove possible to detect such a non-specific surface activity due to divalent cations by observing the effect of adding $\mathrm{Mg}^{++}$to capacitated spermatozoa on fluorescent probe emissions or on the distribution of intramembranous particles.

What mechanisms might be responsible for the specific $\mathrm{Ca}^{++}$effect? In many systems involving fusion of intracellular organelles with the surface membrane a microfilamentous-type contractile system has been implicated. The system is stimulated by $\mathrm{Ca}^{++}$, for which $\mathrm{Ba}^{++}$or $\mathrm{Sr}^{++}$but not $\mathrm{Mg}^{++}$can substitute (Allison, 1973). If actin-like material were present between the acrosomal and plasmalemmal membranes, its contraction on activation could allow the membranes to come into apposition. In preliminary studies, the ability of cytochalasin $\mathrm{B}$ to block activation resulting from addition of $\mathrm{Ca}^{++}$to capacitated spermatozoa has been shown not to prevent activation up to concentrations as high as $100 \mu \mathrm{g} / \mathrm{ml}$. A second hypothesis results from the identification by Yanagimachi \& Usui (1974) of a $\mathrm{Mg}^{++}$-dependent ATPase, active at $\mathrm{pH} \mathrm{7.4,}$ on the outer surface of the acrosomal membrane and the inner surface of the plasma membrane in the guinea-pig (but see Gordon, 1973). The ATPase is inhibited by $\mathrm{Ca}^{++}$. Yanagimachi suggests that perhaps the ATPase affects the osmotic or metabolic regulation in the acrosomal region and that its inhibition by $\mathrm{Ca}^{++}$leads to osmotic swelling of the acrosome with the approximation and fusion of membranes. If this hypothesis in its simplest form is correct, it is testable, for metabolic inhibitors should be capable of inducing the acrosome reaction in spermatozoa capacitated in $\mathrm{Ca}^{++}$-free or in $\mathrm{Mg}^{++}$-containing media. Conversely, $\mathrm{Mg}^{++}$would be expected to inhibit the $\mathrm{Ca}^{++}$activation and this has been shown to be the case. Yet a third hypothesis suggests that a selective permeability to $\mathrm{Ca}^{++}$or perhaps an increased pumping of $\mathrm{Ca}^{++}$into the intermembranous space will result in reduced mutual repulsion of adjacent 
membranes with subsequent fusion (Gordon, 1973). This hypothesis has the required specificity for $\mathrm{Ca}^{++}$, but would be distinguishable from that of Yanagimachi \& Usui (1974) by the differing effects of $\mathrm{Mg}^{++}$and metabolic inhibitors on the process.

The effect of $\mathrm{Ca}^{++}$on the motility of spermatozoa also remains to be explained. This must function independently of the events at the acrosome since even headless spermatozoa will show the whiplash movement. There are clearly many questions remaining to be answered. The acrosome reaction bears similarities with a number of other processes involving exocytosis and endocytosis in diverse tissues (Poste \& Allison, 1973). Experimental designs calculated to explore parallels might enable the acrosome reaction to constitute a component of a more unifying concept of organelle-surface membrane fusion.

\section{ATTACHMENT}

Following the acrosome reaction, a new membrane surface is exposed about which we know little. In the guinea-pig, it is well established that the internal acrosomal membranes are associated with at least two specific autoantigens (Toullet \& Voisin, 1974). Within the guinea-pig acrosome there is also a potent Con A binding site. As described earlier, $95 \%$ of epididymal spermatozoa show a thin rim of surface acrosomal plasma membrane staining with fluorescent Con A. After incubation of spermatozoa in media of different ionic compositions for $15 \mathrm{hr}$, their treatment with fluorescent Con A, using incubating medium for washing and mounting, results in a markedly different distribution of staining patterns (see Table 2). In those media permitting activation, large

Table 2. Fluorescent Concanaval in A staining patterns of the acrosomal region of guinea-pig epididymal spermatozoa after different incubations

\begin{tabular}{llccc}
\hline & Capacitation & \multicolumn{3}{c}{$\%$ Spermatozoa showing: } \\
\cline { 3 - 5 } $\begin{array}{c}\text { Incubation } \\
\text { medium }\end{array}$ & $\begin{array}{c}\text { status of } \\
\text { medium }\end{array}$ & $\begin{array}{c}\text { Thin rim } \\
\text { surface } \\
\text { staining }\end{array}$ & $\begin{array}{c}\text { Heavy } \\
\text { 'bubbly' }\end{array}$ & $\begin{array}{c}\text { No stain } \\
\text { (acrosome } \\
\text { lost) }\end{array}$ \\
\hline No incubation & - & 92 & 3 & 5 \\
$\mathrm{Ca}^{++}$free & Negative & 58 & 40 & 2 \\
$\mathrm{Sr}^{++}$rich & Positive & 13 & 56 & 31 \\
$\mathrm{Ca}^{++}$rich & Positive & 9 & 61 & 30 \\
$\mathrm{Mg}^{++}$rich & Negative & 47 & 48 & 5 \\
\hline
\end{tabular}

numbers of spermatozoa show intense 'bubbly' acrosomal staining. Presumably the fluorescent reagent can now gain entry to target glycosidic residues. In contrast, the media which are $\mathrm{Ca}^{++}$-free or $\mathrm{Mg}^{++}$-rich show very many fewer spermatozoa with 'bubbly' staining acrosomes. That they show any presumably reflects a greater fragility of capacitated spermatozoa resulting in disruption during the centrifugation washes. The intense stains seem to appear first at the tip of the acrosome, but this must constitute a rapid phase since relatively few 
spermatozoa can be found in this state. There is independent evidence that the anterior tip of the acrosome might react first (Gordon et al., 1974; Yanagimachi \& Usui, 1974). The precise location of both the Con A binding sites and the autoantigens internally is not clear, and indeed they may not be tightly membrane bound as the antigens at least are readily water soluble. Neither reagent reacts with the residual inner face of the acrosomal membrane left exposed over the nucleus. It is presumably this face which determines the species specificity of interaction between the zona pellucida and the penetrating spermatozoon (Barros, 1968; Hartmann \& Gwatkin, 1971; Yanagimachi, 1972; Hartmann \& Hutchison, 1974), and which also bears the proteolytic enzymes which facilitate passage of the spermatozoon through the zona pellucida by digestion.

\section{CONGLUSIONS}

The surface membrane of the spermatozoon is clearly a complex mosaic structure, not only in terms of macromolecular composition but also functionally. The observations made on relatively simple cells or cell membranes such as the erythrocyte cannot be readily applied to the more complex spermatozoa. Studies of simpler cells, however, have indicated the approaches that are likely to prove useful, and a consideration of sperm function in molecular terms is now entirely feasible.

\section{ACKNOWLEDGMENTS}

The author wishes to thank Dr R. Yanagimachi and Dr M. Edidin for the stimulation of their discussions and for permission to quote work in press, and Cilla Fuller and Janis Ingram-Johnson for their patient preparation of the manuscript. The experimental work was supported by an M.R.G. grant to the author, and by a grant from the Ford Foundation to Professor G. R. Austin.

\section{REFERENGES}

Allison, A. C. (1973) The role of microfilaments and microtubules on cell movement. Endocytosis and exocytosis in locomotion of tissue cells. Ciba Foundation Symposium, New Series, 14, 109-148. Elsevier, Excerpta Medica, North Holland.

Allison, A. C. \& Mallucci, L. (1964) Lysosomes in dividing cells, with special reference to lymphocytes. Lancet, ii, 1371-1373.

Aonuma, S., Mayumi, T., Suzuku, K., Noguchr, T., Iwari, M. \& Okabe, M. (1973) Studies on sperm capacitation. I. Relationship between a guinea-pig sperm-coating antigen and a spermcapacitation phenomenon. F. Reprod. Fert. 35, 425-432.

Austin, C. R. (1975) Membrane fusion events in fertilization. F. Reprod. Fert. 44, 155-166.

BÄCHI, P. \& Howe, C. (1973) Fusion of erythrocytes by Sendai virus studied by electron microscopy. Proc. Soc. exp. Biol. Med. 141, 141-149.

Barros, C. (1968) In vitro capacitation of golden hamster spermatozoa. Anat. Rec. 160, 310.

Barros, C., Berrios, M. \& Herrera, E. (1973) Capacitation in vitro of guinea-pig spermatozoa in a saline solution. 7. Reprod. Fert. 34, 547-549.

Bedford, J. M. (1963) Morphological reaction of spermatozoa in the female reproductive tract of the rabbit. J. Reprod. Fert. 6, 245-255.

Bedford, J. M. (1970) Sperm capacitation in mammals. Biol. Reprod., Suppl. 2, 128-158.

Bedford, J. M. \& Chang, M. G. (1962) Removal of decapacitation factor from seminal plasma by high speed centrifugation. Am. F. Physiol. 202, 179-181.

Chang, M. G. (1951) Fertilizing capacity of spermatozoa deposited into the Fallopian tubes. Nature, Lond. 168, 697-698. 
Chang, M. C. (1957) Detrimental effect of seminal plasma on the fertilizing capacity of sperm. Nature, Lond. 179, 258-259.

Chapman, D. (1972) Nuclear magnetic resonance spectroscopic studies of biological membranes. Ann. N.Y. Acad. Sci. 195, 179-186.

Cooper, G. W. \& BEDFord, J. M. (1971) Acquisition of surface charge by the plasma membrane of mammalian spermatozoa during epididymal maturation. Anat. Rec. 169, 300-301.

Devaux, P. \& MaConneld, H. M. (1972) Lateral diffusion in spin-labelled phosphatidylcholine multilayers. J. Am. chem. Soc. 94, 4475-4481.

Dukelow, W. R., Ghernoff, H. N., Pinsker, M. C. \& Williams, W. L. (1966) Enzymatic activities at the time of sperm capacitation. F. Dairy Sci. 49, 725.

Dukelow, W. R., Chernoff, H. N. \& Williams, W. L. (1966) Enzymatic characterisation of decapacitation factor. Proc. Soc. exp. Biol. Med. 121, 396-398.

Dukelow, W. R., Chernoff, H. N. \& Williams, W. L. (1967a) Fertilizable life of the rabbit ovum relative to sperm capacitation. Am. J. Physiol. 213, 1397-1400.

Dukelow, W. R., Chernoff, H. N. \& Williams, W. L. (1967b) Properties of decapacitation factor and presence in various species. 7. Reprod. Fert. 14, 393-399.

Eoflman, G. M. \& Millette, G. F. (1971) Molecular probes of spermatozoan structure. Proc. natn. Acad. Sci. U.S.A. 68, 2436-2440.

Edelman, G. M., Yahara, I. \& Wang, J. L. (1973) Receptor mobility and receptor-cytoplasmic interactions in lymphocytes. Proc. natn. Acad. Sci. U.S.A., 70, 1442-1446.

Eoroin, M. (1974) Rotational and translational diffusion in membranes. Ann. Res. Biophys. biol. Eng. 3, $179-202$.

Ericsson, R. J. (1967) A fluorometric method for measurement of sperm capacitation. Proc. Soc. exp. Biol. Med. 125, 1115-1118.

Ericsson, R. J., Buthala, B. A. \& Norland, J. F. (1971) Fertilization of rabbit ova in vitro by sperm with adsorbed Sendai virus. Science, $\mathcal{N} . \Upsilon$. 173, 54-55.

FRYe, L. D. \& Edidin, M. (1970) The rapid intermixing of cell surface antigens after formation of mouse-human heterokaryons. F. Cell Sci. 7, 319-335.

Gilula, N. B., Branton, C. \& SATIR, P. (1970) The septate junction: a structural basis for intercellular coupling. Proc, natn. Acad. Sci. U.S.A. 67, 213-220.

Gordon, M. (1973) Localization of phosphatase activity on the membranes of the mammalian sperm head. F. exp. Zool. 185, $111-123$.

Gordon, M., Dandekar, P. V. \& Bartoszewicz, W. (1974) Ultrastructural localization of surface receptors for Concanavalin A on rabbit spermatozoa. 7. Reprod. Fert. 36, 211-214.

Gwatkin, R. B. L. \& Hutchison, C. F. (1971) Capacitation of hamster spermatozoa by $\beta$-glucuronidase. Nature, Lond. 229, 343-344.

Hamner, C. E., Jones, J. P. \& Sojka, N. J. (1968) Influence of hormonal state of the female on the fertilizing capacity of rabbit spermatozoa. Fert. Steril. 19, 137-143.

Hartmann, J. F. \& Gwatkin, R. B. L. (1971) Alteration of sites on the mammalian sperm surface following capacitation. Nature, Lond. 234, 479-481.

Hartmann, J. F. \& Hutchison, C. F. (1974) Contact between hamster spermatozoa and the zona pellucida releases a factor which influences early binding stages. 7 . Reprod. Fert. 37, 61-66.

Hirschhorn, R., Brittinger, G., Hirschhorn, K. \& Weissmann, G. (1968) Studies on lysosomes. XII. Redistribution of acid hydrolases in human lymphocytes stimulated by phytohaemaglutinin. 7. Cell Biol. 37, 412-423.

Howeld, J. I., Ahkong, Q. F., Cramp, F. C., Fisher, D., Tampion, W. \& Lucy, J. A. (1972) The fusion of hen erythrocytes by non-ionic surface-active adjuvants. Biochem. $7.130,44 \mathrm{P}-45 \mathrm{P}$.

Hsia, J. C., Piette, L. H. \& Noyes, R. W. (1969) Binding of spermatozoa with surface active spinlabels. F. Reprod. Fert. 20, 147-149.

Hunter, A. G. \& Nornes, H. O. (1969) Characterization and isolation of a sperm coating antigen from rabbits seminal plasma with a capacity to block fertilization. 7. Reprod. Fert. 20, 419-427.

INBAR, M., BEN-BASSAT, H. \& SACHS, L. (1973) Temperature-sensitive activity on the surface membrane in the activation of lymphocytes by lectins. Expl Cell Res. 76, 143-151.

Johnson, M. H. (1970) Selective damage to spermatogenic cells of high antigenicity during autoallergic aspermatogenesis. 7. Path. Bact. 102, 131-138.

Johnson, M. H. (1975) Antigens of peri-implantation trophoblast. In Immunobiology of Trophoblast, pp. 87-100. Eds. R. G. Edwards, C. W. S. Howe and M. H. Johnson. Cambridge University Press.

Johnson, M. H. \& Edidin, M. (1972) H-2 antigens on mouse spermatozoa. Transplantation, 14, 781786.

Johnson, M. H. \& Howe, C. W. S. (1975) The immunobiology of spermatozoa. Proc. Linn. Soc. Lond. (in press).

Johnson, W. L. \& Hunter, A. G. (1972a) Immunofluorescent evaluation of the male rabbit reproductive tract for sites of secretion and absorption of seminal antigens. Biol. Reprod. 6, 13-22. 
Johnson, W. L. \& Hunter, A. G. (1972b) Seminal antigens: their alteration in the genital tract of female rabbits and during partial in vitro capacitation with $\beta$-amylase and $\beta$-glucuronidase. Biol. Reprod. 7, 332-340.

Joshi, M. S. \& Murray, I. M. (1974) Immunological studies of the rat uterine fluid peptidase. 7 . Reprod. Fert. 37, 361-365.

Joshi, M. S., YARon, A. \& Lindner, H. R. (1970) An endopeptidase in the uterine secretion of the preoestrous rat and its relation to a sperm decapacitating factor. Biochem. biophys. Res. Commun. 38, 52-57.

Killian, G. J. \& Amann, R. P. (1973) Immunoelectrophoretic characterization of fluid and sperm entering and leaving the bovine epididymis. Biol. Reprod. 9, 489-499.

KIRTon, K. T. \& HAFs, H. D. (1965) Sperm capacitation by uterine fluid or $\beta$-amylase in vitro. Science, N.Y. 150, 618-619.

Koemler, J. K. \& Perkins, W. D. (1974) Fine structure observations on the distribution of antigenic sites on guinea-pig spermatozoa. F. Cell Biol. 60, 789-795.

König, E., BRITtinger, G. \& Cohnen, G. (1973) Relation of lysosomal fragility in CLL lymphocytes to PHA reactivity. Nature, New Biol. 244, 247-248.

Koo, G. G., Stackpole, C. W., Boyse, E. A., Hammerling, U. \& Lardis, M. P. (1973) Topographical location of $\mathrm{H}-\mathrm{Y}$ antigen on mouse spermatozoa by immunoelectronmicroscopy. Proc. natn. Acad. Sci. U.S.A. 70, 1502-1505.

LeVine, N. \& MARSH, D. J. (1971) Micropuncture studies of the electrochemical aspects of fluid and electrolyte transport in individual seminiferous tubules, the epididymis and the vas deferens in rats. F. Physiol., Lond. 213, 557-570.

LiNDAHL, D. E. (1973) Activators of the ATP-dependent surface reaction in apical cell membrane of the bull-sperm head causing head-head association. Expl Cell Res. 81, 413-431.

Linden, C. D., Wright, K. L., MaConneli, H. M. \& Fox, C. F. (1973) Lateral phase separations in membrane lipids and the mechanism of sugar transport in Escherichia coli. Proc. natn. Acad. Sci. U.S.A. 70, 2271-2275.

Lucy, J. A. (1975) Aspects of the fusion of cells in vitro without viruses. F. Reprod. Fert. 44, 193-205.

Machtiger, N. A. \& Fox, C. F. (1973) Biochemistry of bacterial membranes. A. Rev. Biochem. 42, 575600.

MaHi, C. A. \& Yanagimachi, R. (1973) The effects of temperature osmolality and hydrogen ion concentration on the activation and acrosome reaction of golden hamster spermatozoa. F. Reprod. Fert. $35,55-66$.

Mercado, E. \& Rosado, A. (1973) Structural properties of the membrane of intact human spermatozoa. A study with fluorescent probes. Biochim. biophys. Acta, 298, 639-652.

Mryamoto, H. \& Chang, M. C. (1973) Effect of osmolality on fertilization of mouse and golden hamster eggs in vitro. 7 . Reprod. Fert. 33, 481-487.

Munro, A. J. (1975) Antigenic topography and the interaction of antibodies and immune cells with surface antigens. In Immunobiology of Trophoblast, pp. 5-12. Ed. R. G. Edwards, C. W. S. Howe and M. H. Johnson. Cambridge University Press.

NickLAs, W. J. \& BerL, S. (1974) Effects of cytochalasin B on uptake and release of putative transmitters by synaptosomes on brain actomycin-like proteins. Nature, Lond. 247, 471-473.

Nicolson, G. L. (1972) Topograph of Concanavalin A sites modified by proteolysis. Nature, New Biol. 239, 193-196.

Nigolson, G. L. \& Yanagimachi, R. (1972) Terminal saccharides on sperm plasma membranes: identification using specific agglutinins. Science, N.Y. 177, 276-279.

Nicolson, G. L. \& YanagrmaGhi, R. (1974) Mobility and the restriction of mobility of plasma membrane lectin-binding components. Science, N.X. 184, 1294-1296.

Noyes, R. W., Walton, A. \& Adams, C. E. (1958) Capacitation of rabbit spermatozoa. 7. Endocr. 7 , 374-380.

Oliphant, G. \& Brackett, B. G. (1973a) Immunological assessment of surface changes of rabbit's sperm undergoing capacitation. Biol. Reprod. 9, 404-414.

Oliphant, G. \& BRACKetT, G. B. (1973b) Capacitation of mouse spermatozoa in media with elevated ionic strength and reversible decapacitation with epididymal extracts. Fert. Steril. 24, 948-955.

Overath, P., Hill, F. F. \& Lamneh-Hrrsch, I. (1971) Biogenesis of $E$. coli membrane: evidence for randomization of lipid phase. Nature, New Biol. 234, 264-267.

Papahedjopoulos, D., Poste, G. \& Schaeffer, B. (1973) Fusion of mammalian cells by unilamellar lipid vesicles: influence of lipid surface charge, fluidity and cholesterol. Biochim. biophys. Acta, 323, $23-40$.

Petrt, V. A. \& Edrdin, M. (1974) Lateral phase separations of lipids in mammalian plasma membranes: the effect of temperature on the mobility of membrane antigens. Science, $\mathcal{N} . X .134,1183-1185$.

Pinsker, M. G. \& Williams, W. L. (1967) Properties of a spermatozoa anti-fertility factor. Archs Biochem. Biophys. 122, $111-117$. 
Poste, G. \& Alurson, A. C. (1973) Membrane fusion. Biochim. biophys. Acta, 300, 421-465.

Robertson, R. T., Bhalla, V. K. \& Williams, W. L. (1971) Purification and the peptide nature of decapacitation factor. Biochem. biophys. Res. Commun. 45, 1331-1336.

Rosado, A., Velazquez, A. \& Lara-Ricalde, R. (1973) Gell polarography. II. Effect of neuraminidase and follicular fluid upon the surface characteristics of human spermatozoa. Fert. Steril. 24, 349-354.

SAtir, B., Schooley, C. \& SATIR, P. (1972) Membrane reorganisation during secretion in Tetrahymena. Nature, Lond. 235, 53-54.

SAttr, B., Schooley, C. \& SAtir, P. (1973) Membrane fusion in a model system. F. Cell Biol. 56, 153-176.

Scandell, G. J., Deveaux, P. \& McConnell, H. M. (1972) Rapid lateral diffusion of phospholipid in rabbit cytoplasmic reticulum. Proc. natn. Acad. Sci. U.S.A. 69, 2056-2060.

Scotr, T. W., Voglmayr, J. K. \& Setchell, B. P. (1967) Lipid composition and metabolism in testicular and ejaculated ram spermatozoa. Biochem. F. 102, 456-461.

Shimshick, E. J. \& MCConneLl, H. M. (1973) Lateral phase separations in binary mixtures of cholesterol and phospholipids. Biochem. biophys. Res. Commun. 53, 464-451.

Singer, S. J. (1971) The molecular organization of biological membranes. In Structure and Function of Biological Membrane, pp. 146-224. Ed. L. I. Rothfield. Academic Press, New York.

Singer, S. J. \& Nicoison, G. L. (1972) The fluid mosaic model of the structure of cell membranes. Science, N.Y. 175, 720-731.

Smith, H., Ryan, J. W. \& Smith, D. S. (1973) Freeze-etch studies on the plasma membrane of pulmonary endothelial cells. F. Cell Biol. 56, 492-499.

Soupart, P. (1967) Studies on the hormonal control of rabbit sperm capacitation. 7. Reprod. Fert., Suppl. 2, 49-64.

Suominen, J. \& Setchell, B. P. (1972) Enzymes and trypsin inhibitor in the rete testis fluid of rams and boars. F. Reprod. Fert. 30, 235-245.

Toullet, F. \& Vorsin, G. A. (1974) Spermatotoxic, sperm agglutinating and cytotoxic activities of guinea-pig autoantibodies to sperm autoantigen T. F. Reprod. Fert. 37, 299-313.

Tourtellotte, M. E., Branton, D. \& Keith, A. (1970) Membrane structure: spin-labelling and freeze etching of Mycoplasma laidlawii. Proc. natn. Acad. Sci. U.S.A. 66, 909-916.

TRÄUBLE, H. \& EIBL, H. (1974) Electrostatic effects on lipid phase transitions: membrane structure and ionic environment. Proc. natn. Acad. Sci. U.S.A. 71, 214-219.

TRÄUble, H. \& SACKMANN, E. (1972) Studies of the crystalline-liquid crystalline phase transition of lipid model membranes. III. Structure of a steroid-lecithin system below and above the lipid-phase transition. F. Am. chem. Soc. 94, 4499-4510.

UKENA, T. E. \& BERLIN, R. D. (1972) Effects of colchicine and vinblastine on the topographical separation of membrane functions. F. exp. Med. 136, 1-7.

VAIDYA, R. A., Bedford, J. M., Glass, R. H. \& Morris, J. (1969) Evaluation of the removal of tetracycline fluorescence from spermatozoa as a test for capacitation in the rabbit. F. Reprod. Fert. 19, 483-489.

Vaidya, R. A., Glass, R. H., Dandekar, P. \& Johnson, K. (1971) Decrease in the electrophoretic mobility of rabbit spermatozoa following intrauterine incubation. F. Reprod. Fert. 24, 299-301.

Werl, A. J. \& RoDENBURG, J. M. (1962) The seminal vesicle as the source of the spermatozoa-coating antigen of seminal plasma. Proc. Soc. exp. Biol. Med. 109, 567-570.

Weinman, D. E. \& Williams, W. L. (1964) Mechanism of capacitation of rabbit spermatozoa. Nature, $203,423-424$.

Wisnieski, B. J., Williams, R. E. \& Fox, C. F. (1973) Manipulation of fatty acid composition in animal cells grown in culture. Proc. natn. Acad. Sci. U.S.A. 70, 3669-3673.

Woobing, F. B. P. (1975) Studies on the mechanism of the hyamine-induced acrosome reaction in ejaculated bull spermatozoa. F. Reprod. Fert. 44, 185-192.

YANAGIMACHI, R. (1969) In vitro capacitation of hamster spermatozoa by follicular fluid. F. Reprod. Fert. $18,275-286$.

Yanagimachi, R. (1972) Penetration of guinea-pig spermatozoa into hamster eggs in vitro. f. Reprod. Fert. 28, 477-480.

Yanagimachi, R., Nicolson, G. L., Noda, Y. D. \& Fujimoto, M. (1973) Electron microscopic observations of the distribution of acidic anionic residues on hamster spermatozoa and eggs before and during fertilization. F. Ultrastruct. Res. 43, 344-353.

Yanagimachi, R., Noda, Y. D., Fujimoto, M. \& Nicolson, G. L. (1972) The distribution of negative surface charges on mammalian spermatozoa. Am. F. Anat. 135, 497-520.

YANAGMachi, R. \& Usui, N. (1974) Calcium dependence of the acrosome reaction and activation of guinea pig spermatozoa. Expl Cell Res. 89, 161-174.

Zaneveld, L. J. D. \& Williams, W. L. (1970) A sperm enzyme that disperses the corona radiata and its inhibition by decapacitation factor. Biol. Reprod. 2, 363-368. 